\title{
Cross Sectional Study on a Spectrum of Bacterial Pathogens under Actions of Infection Control in Different Clinical Specimens of ICU Patients of Sajer General Hospital in Saudi Arabia, and Their Antibiotic Sensitivity Pattern
}

\author{
Atallah Alenezi ${ }^{1}$, Noha El-Tahtawi ${ }^{2}$, Entesar Mahmoud Makhlouf ${ }^{3}$
}

1Department of Nursing Microbiology, Mental Health Nursing, College of Applied Medical Sciences, Shaqra University, Saudi Arabia. ${ }^{2}$ Department of Biology, College of Science and Art, Shaqra University, Saudi Arabia. ${ }^{3}$ Department of Maternity, Obstetrics and Gynaecological Nursing, College of Applied Medical Sciences, Shaqra University (KSA).

\section{ABSTRACT}

\section{BACKGROUND}

The intensive care unit (ICU) is a special department of the hospital and health care setting that provides intensive nursing care and care to patients with the most severe and life-threatening illnesses and injuries that require accurate, continuous monitoring and support from specialist, medication, and equipment in order to maintain normal bodily functions. Nosocomial infections are one of the leading causes of mortality in hospitalised patients especially the critically ill patients in the intensive care unit. The purpose of the present study was isolation, identification, and detection of the antibiotic sensitivity pattern of pathogenic bacteria isolated from different clinical specimens of ICU patients under the effect of infection control in Sajer General Hospital in Saudi Arabia.

\section{METHODS}

In this cross-sectional study conducted from January 2020 to April 2020, 200 clinical samples, 40 blood, 45 urine, 50 tracheal aspirate, 30 post-surgical Caesarean section wound swabs, 20 central venous catheters and 15 pus swabs were collected from patients hospitalised in ICUs of Sajer General Hospital. Antibiotic sensitivity testing was performed with the diffusion-disk method for several antibiotics.

\section{RESULTS}

The rate of nosocomial infection among ICU patients due to Gram-negative bacteria is significantly higher than that recorded by Gram-positive bacteria (78.75 \%, 21.25 $\%$, respectively). The majority of frequent bacteria isolated from all clinical specimens were Klebsielleae pneumoniae 20 (25\%) followed by Pseudomonas aeruginosa 18 (22.5\%), Acinetobacter baumannii 15 (18.75\%), Escherichia coli 10 (12.50\%), Staphylococcus aureus 9 (11.25\%), Streptococcus pyogenes 6 (7.50\%) and enterococcus spp. 2 (2.50\%). K. pneumoniae, P. aeruginosa and E. coli achieved high sensitivity to imipenem and meropenem (100\%). A. baumannii showed high sensitivity to meropenem (100\%) and imipenem (93.33\%). Staph. aureus, Streptococcus pyogenes and enterococcus species showed high sensitivity to vancomycin $(100 \%)$.

\section{CONCLUSIONS}

The nosocomial infection is a major cause of mortality and morbidity in hospitals. The role of nursing care in the prevention of nosocomial infection has proven vital due to the emergence and spreading of different pathogenic bacteria. Close interactions between health care providers can save many more lives.

\section{KEY WORDS}

Intensive Care Units, Antibiotics Sensitivity Pattern, Nursing Infection Control
Corresponding Author: Dr. Entesar Makhlouf, Department of Nursing - Microbiology Maternity, Obstetrics \& Gynaecological Nursing, Assiut University, Egypt. E-mail: e.makhlouf@su.edu.sa

DOI: $10.14260 /$ jemds/2021/761

How to Cite This Article:

Alenezi A, El-Tahtawi N, Makhlouf EM. Cross sectional study on a spectrum of bacterial pathogens under actions of infection control in different clinical specimens of ICU patients of Sajer general hospital in Saudi Arabia, and their antibiotic sensitivity pattern. J Evolution Med Dent Sci 2021;10(44):3762-3768, DOI: 10.14260/jemds/2021/761

Submission 25-01-2021, Peer Review 01-11-2021, Acceptance 08-11-2021, Published 30-11-2021.

Copyright (C) 2021 Atallah Alenezi et al. This is an open access article distributed under Creative Commons Attribution License [Attribution 4.0 International (CC BY 4.0)] 


\section{BACKGROUND}

Infection prevention and control interventions have been applied in different hospitals and health care settings to decrease and prevent the growth, development and spreading of harmful pathogenic bacteria in adult intensive care units (ICUs). The interventions vary widely according to region and effect. ${ }^{1}$ The Centers for Disease Control and Prevention, Healthcare Infection Control Practices Advisory Committee published guidelines for the management of several pathogenic bacteria at 2006 and 2007 and using of different precautions in healthcare settings. ${ }^{2,3}$ The European Society of Clinical Microbiology and Infectious Diseases published important guidelines for lowering, prevention and control of different pathogenic bacteria in different hospitalized patients in 2013.4 Despite several recommendations and publishers over the years, the relative efficacy of infection prevention control interventions are still in conclusive, especially in highresistance endemic settings. ${ }^{5}$

Great efforts to control intensive care unit infections (ICUIs) with few exceptions have failed, resulting in growing disturbance in the medical field and society. The rate of mortality increases due to spreading of antibiotic-resistant bacteria in a hospital in all over the world. ${ }^{6}$ The European Commission published a Council Recommendation on patient safety, including the control and prevention of ICUs in hospitals in January 2009.7

ICU is the important department in both hospital and health care setting which provides intensive nursing care and care to patients with the most severe and life-threatening illnesses and injuries. Intensive care units staffed by highly trained doctors and nurses who specialize in caring for seriously ill-patients. Patients may be transferred from a ward if they rapidly deteriorate, from an emergency department if required or immediately after surgery if the surgery is majorly invasive and if the patient is at high risk of complications to an (ICU) ${ }^{8}$. Most patients in intensive care units are exposed to nosocomial infections, this infections are not present at the time of the admission to hospital and appear within 48 to 72 hours after admission or within 10 days after discharge. 9 Although patients in the ICU consists of $5-10 \%$ of total patients in the hospital, the infection rates in the ICU are very high and range from 15 to $50 \%$ and are highest in burn and surgical ICUs and the lowest in coronary units. ${ }^{10}$ This related to the usage of large number of invasive procedures and devices, such as tracheotomy tubes and endotracheal, other risk factors that contribute to susceptibility of patients to infection include, immune compromised state, age, malnutrition and severe underlying diseases and high incidence of cross infection. ${ }^{11}$ Ventilator-associated pneumonias, urinary tract infection (UTIs) and blood stream infections are very common in ICU. ${ }^{12}$ The ICU environment of the hospital is considered as a focus of the emergence and spreading of several pathogenic bacteria. ${ }^{13}$

Spreading of infections with different strains of resistant pathogenic bacteria is considered as one of the leading causes of mortality in hospitalized patients in the intensive care unit. ${ }^{14}$ The types of pathogenic bacteria causing infections and their antibiotic resistance pattern vary widely from one hospital to another. 15 The study in an Indian ICU revealed that the most common bacteria were Escherichia coli, Pseudomonas aeruginosa, Staphylococcus aureus, proteus spp, and klebsiella spp. ${ }^{16}$ In a European ICU, Staphylococcus aureus was found as the most frequently isolated organism (30.1\%) followed by Pseudomonas aeruginosa (28.7 \%), coagulase- negative staphylococcus $(19.1 \%){ }^{17}$

Many ICUs in the world are suffering from increasingly rapid emergence and spread of antibiotic-resistant bacteria because of the high use of broad-spectrum antibiotics in specialized areas of the hospital, crowding of patients with high levels of disease acuity in relatively small, shortage of nursing and other supporting staff due to economic pressures and the presence of many acutely and chronically ill patients who require prolonged hospitalization. ${ }^{18,19}$

Risk factors like indiscriminate and inadequately prolonged use of antibiotics also leads to the emergence and proliferation of resistant strains preferentially, prescription of antibiotics prophylactically and empirically without carrying out sensitivity tests particularly in developing countries leading to increase of resistant pathogenic bacteria. In intensive care units, patients may be immune-compromised, and many prosthetics and instrumentations are used routinely. ${ }^{20}$

Prevention of the emergence and dissemination of resistant bacteria will reduce the spreading of infectious diseases in ICUs. The appropriate antibiotic application that includes optimal selection, dose, and duration of treatment, as well as control of antibiotic use, will slow or prevent the emergence of resistance among bacteria. ${ }^{21}$ Monitoring the use of different antibiotics and review of sensitivity patterns is imperative. Audit of antibiotic sensitivity patterns in ICUs and critical care units is crucial and far more important for giving effective treatment and decreasing the spread of resistant bacteria. ${ }^{22}$

The purpose of the present study was isolation, identification, and detection of the antibiotic sensitivity pattern of pathogenic bacteria isolated from different clinical specimens of ICU patients under the effect of infection control in Sajer, General Hospital in Saudi Arabia.

\section{METHODS}

The present work is a descriptive cross-sectional study. In a 3month period from June to August 2020, 200 clinical specimens were taken from all 100 patients staying longer than $48 \mathrm{~h}$ in the ICU of Sajer, General Hospital in Saudi Arabia. The microbiological analysis was carried out at microbiology laboratory for isolation of pathogenic bacteria that cause nosocomial infections in ICU.

Two hundred clinical samples include (40 blood, 45 urine, 50 tracheal aspirate, 30 post Caesarean section surgical wounds swabs, 20 central venous catheters and 15 pus swabs) were collected from the patients who were admitted to the ICUs and confirmed as nosocomial infected. The time gap between two samples from each patient was five days. There was no basis for the number of different samples of urine, blood etc. to be collected in this study, as it was only available samples. 


\section{Cultivation and Identification}

All clinical specimens except urine specimens were aseptically inoculated on plates of nutrient agar, MacConkey's agar and blood agar whereas urine samples were inoculated on plates of cystine-lactose-electrolyte-deficient (CLED) agar and nutrient agar (Oxoid Cambridge, UK). All inoculated plates were incubated at $37^{\circ} \mathrm{C}$ for $72 \mathrm{~h}$. The identification of bacteria was done by macro and micro morphological evaluation. The characteristics considered were size, shape, colour, pigmentation and haemolytic nature of colonies. Also, biochemical tests were applied by using conventional methods and API strips (20NE and 10S).

\section{Antibiotic Sensitivity Testing}

An antibiotic sensitivity test was detected by using modified Kirby-Bauer Disk diffusion method using Muller-Hinton agar and results were determined according to the CLSI, 2009. From bacterial pure culture 2 - 3, selected colonies of bacteria were taken with a sterile cotton swab and transferred to a tube containing peptone water, mixed well and incubated at room temperature for $30 \mathrm{~min}$. Then swab was taken, and the excess suspension was removed gently by rotation of the swab against the surface of the test tube. The inoculated plates were then left at room temperature to dry for $2-4 \mathrm{~min}$ and antibiotic discs were placed on the surface of Muller-Hinton agar plate. Twelve antibiotics were used in this study ampicillin, amoxycillin, amikacin, azithromycin, ciprofloxacin, ceftazidime, cefoperazone, gentamicin, imipenem, meropenem, ofloxacin and vancomycin.

\section{Statistical Analysis}

The information taken was coded, analysed and arranged. Descriptive analysis was done in this research, including frequencies and rate, additionally utilising the mean, standard deviation, t-test. It was analysed by the SPSS version 20 .

\section{Ethical Considerations}

Before directing this research, ethical clearance was taken from Sajer, General Hospital in Saudi Arabia. There were no risks that could affect the women during the application of the study. Informed consent was acquired, and the relatives of women were rest assured of namelessness and confidentiality. No harm was foreseen.

\section{RESULTS}

In the 3 months study period, total 200 clinical samples from 100 patients in ICU were collected and studied. The collected isolates of all samples together were 80 (40\%). There were 63 Gram-negative bacteria accounting for $78.75 \%$ and 17 Grampositive bacteria accounting for (21.25\%) Figure 1.

Out of these, 30 isolates $(37.5 \%)$ were from tracheal aspirate; 20 (25\%) isolates from urine; 11 (13.75\%) isolates from blood samples; 9 (11.25\%) isolates from post Caesarean section surgical wound swabs; $6(7.5 \%)$ isolates from central venous catheters and 4 (5\%) isolates from pus swabs as shown in Table 1.
Out of these isolates the most prevalent bacterial organism from all clinical specimens was Klebsielleae pneumoniae 20 (25 \%) followed by Pseudomonas aeruginosa 18 (22.5 \%), Acinetobacter baumannii 15 (18.75\%), Escherichia coli 10 (12.50\%), Staphylococcus aureus 9 (11.25\%), Streptococcus pyogenes $6(7.50 \%)$ and enterococcus spp. 2 (2.50\%). as shown in Table 1, Figure 2.

K. pneumonia (40\%), A. baumannii (23.33\%) and $P$. aeruginosa $(16.66 \%)$ were the commonest organisms isolated from tracheal aspirate. E. coli (40\%) and K. pneumonia (25\%) were the most prevalent organisms isolated from urine samples. Streptococcus pyogenes (36.36\%) and Staphylococcus aureus (27.27 \%) were the most frequently isolated organisms from blood samples. The most common organism isolated from post Caesarean section surgical wound swabs and pus swabs was Pseudomonas aeruginosa with percentage incidence (55.55 \% and $50 \%$ respectively). Whereas the bacterial organisms (Streptococcus pyogenes, Staphylococcus aureus, Acinetobacter baumannii and Pseudomonas aeruginosa) which were isolated from central venous catheter tips achieved the same percentage incidence $(25 \%)$. as shown in pneumonia isolates showed high, moderate and low sensitivity rates against all antibiotics tested. The most effective antibiotics were imipenem and meropenem, the sensitivity of K. pneumoniae isolates was $100 \%$, followed by ciprofloxacin, cefoperazone ofloxacin, amikacin and gentamicin, the susceptibility of $K$. pneumonia was $(95 \%, 90$ $\%, 85 \%, 75 \%$ and $50 \%$, respectively). Few isolates of $K$. Pneumonias hovered very low sensitivity rates with amoxicillin, ceftazidime and ampicillin (20\%, $15 \%$ and $10 \%$, respectively).

Pseudomonas aeruginosa showed moderate to high sensitivity rates against six types of antibiotics. Isolates of $P$. aeruginosa were very sensitive to three types of antibiotics, imipenem, meropenem and gentamicin with $100 \%$ susceptibility rates. The effect of amikacin, ofloxacin and ciprofloxacin against some isolates of $P$. aeruginosa was moderate, the sensitivity of these isolates against these antibiotics was (88\%, $83.33 \%$ and $77.7 \%$, respectively). Isolates of $P$. aeruginosa were resistant to ampicillin, amoxicillin, ceftazidime and cefoperazone.

Most isolates of Acinetobacter baumannii showed moderate to high sensitivity rates against different types of antibiotics. Acinetobacter baumannii showed high sensitivity (100\%) to four antibiotics, amoxicillin, gentamicin, meropenem and ofloxacin, followed by ciprofloxacin, imipenem and ampicillin with sensitivity rates (93.33\%, 93.33 $\%$ and $86.66 \%$, respectively). A. baumannii exhibited moderate sensitivity to ceftazidime $(66.66 \%)$ and low sensitivity to amikacin (13.33\%). Escherichia coli was very sensitive to imipenem (100\%), meropenem (100\%), gentamicin (90\%), amikacin (90.0\%), ofloxacin (80\%), ciprofloxacin (70\%) and ceftazidime (60\%); but was resistant to ampicillin.

Staphylococcus aureus isolates showed high sensitivity to azithromycin (100\%) cefoperazone (100\%), meropenem (100\%), vancomycin (100\%), amikacin (88.88\%) and ciprofloxacin (77.77 \%). Also, Staphylococcus aureus isolates showed moderate sensitivity to ceftazidime (55.55\%) and gentamicin (44.44\%). Few isolates of $S$. aureus exhibited low sensitivity to amoxicillin (22.22\%) and ofloxacin (11.11\%) but were resistant to ampicillin. 

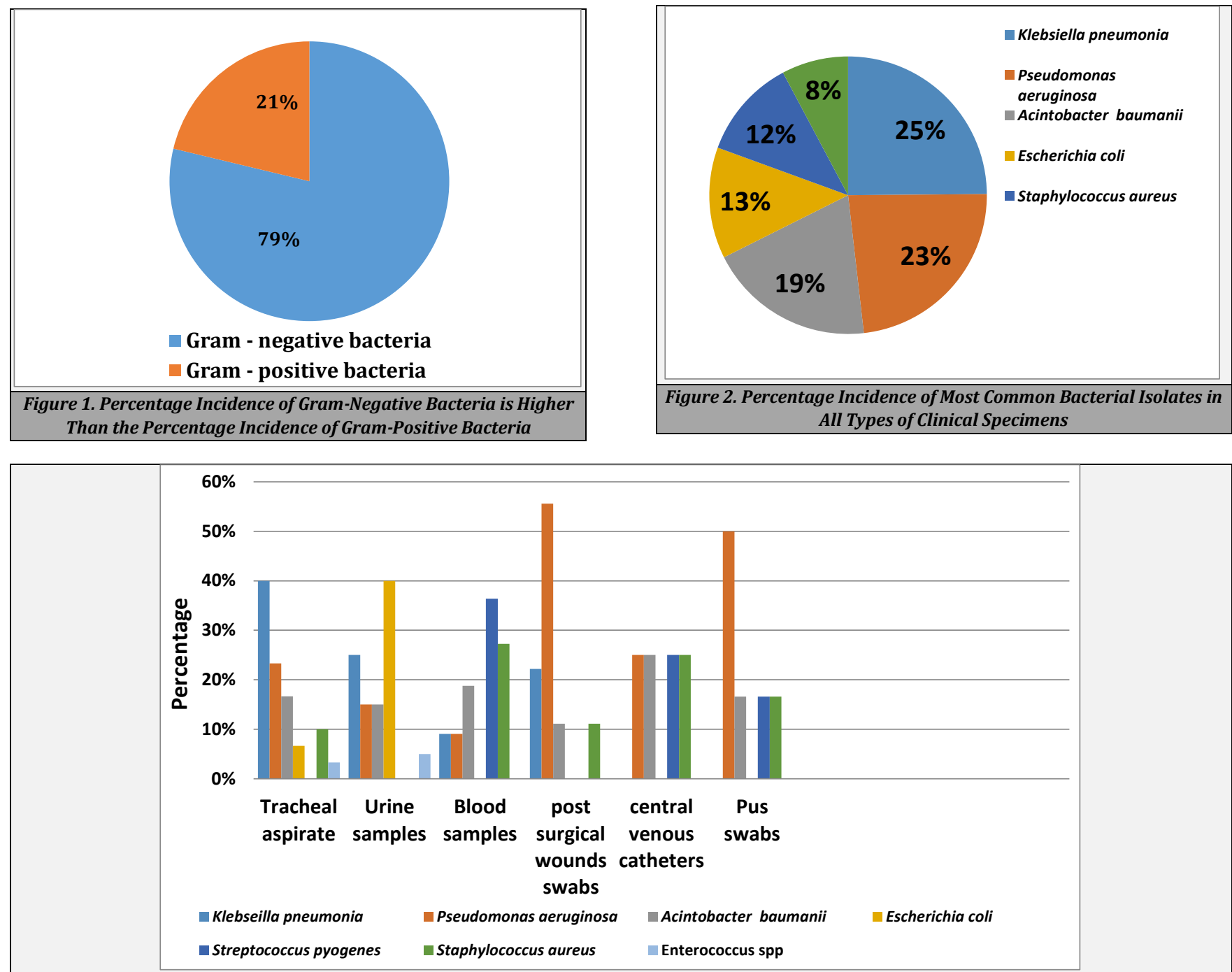

Figure 3. Percentage Incidence of the Most Common Bacterial Species Collected from Different Types of Clinical Specimens of ICU Patients

\begin{tabular}{|c|c|c|c|c|c|c|c|}
\hline \multirow[b]{2}{*}{ Bacterial Isolates } & \multicolumn{7}{|c|}{ Different Clinical Specimen N = 200} \\
\hline & Tracheal Aspirate & Urine Samples & Blood Samples & $\begin{array}{l}\text { Post Caesarean Section } \\
\text { Surgical Wounds Swabs }\end{array}$ & $\begin{array}{l}\text { Central Venous } \\
\text { Catheters }\end{array}$ & $\begin{array}{l}\text { Pus } \\
\text { Swabs }\end{array}$ & Total \\
\hline Klebsiella pneumonia & $12(40 \%)$ & $5(25 \%)$ & $1(9.1 \%)$ & $2(22.22 \%)$ & - & - & 20 \\
\hline Pseudomonas aeruginosa & $5(23.33 \%)$ & $3(15 \%)$ & $(9.1 \%)$ & $5(55.55 \%)$ & $1(25 \%)$ & $3(50 \%)$ & 18 \\
\hline Acinetobacter baumannii & $7(16.66 \%)$ & $3(15 \%)$ & $2(18.8 \%)$ & $1(11.11 \%)$ & $1(25 \%)$ & $1(16.6 \%)$ & 15 \\
\hline Escherichia coli & $2(6.66 \%)$ & $8(40 \%)$ & 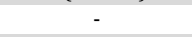 & - & - & $1(16.6 \%)$ & 10 \\
\hline Streptococcus pyogenes & & - & $4(36.36 \%)$ & - & $1(25 \%)$ & $1(16.6 \%)$ & 6 \\
\hline Staphylococcus aureus & $3(10 \%)$ & - & $3(27.27 \%)$ & $1(11.11 \%)$ & $1(25 \%)$ & $1(16.6 \%)$ & 9 \\
\hline Enterococcus spp. & $1(3.3 \%)$ & $1(5 \%)$ & - & - & - & - & 2 \\
\hline Total & 30 & 20 & 11 & 9 & 4 & 6 & 80 \\
\hline
\end{tabular}

\begin{tabular}{|c|c|c|c|c|}
\hline Antibiotics & K. pneumoniae $\mathrm{N}=20$ & P. aeruginosa $\mathrm{N}=18$ & A. baumannii $\mathrm{N}=15$ & E. coli $\mathrm{N}=10$ \\
\hline Ampicillin & $2(10 \%)$ & 0 & $13(86.66 \%)$ & 0 \\
\hline Amoxicillin & $4(20 \%)$ & 0 & $15(100 \%)$ & $2(20 \%)$ \\
\hline Amikacin & $15(75 \%)$ & $16(88 \%)$ & $2(13.33 \%)$ & $9(90 \%)$ \\
\hline Azithromycin & NT & NT & NT & NT \\
\hline Ciprofloxacin & $19(95 \%)$ & $14(77.7 \%)$ & $14(93.33 \%)$ & $7(70 \%)$ \\
\hline Ceftazidime & $3(15 \%)$ & 0 & $10(66.66 \%)$ & $6(60 \%)$ \\
\hline Cefoperazone & $18(90 \%)$ & 0 & NT & NT \\
\hline Gentamicin & $10(50 \%)$ & $18(100 \%)$ & $15(100 \%)$ & $9(90 \%)$ \\
\hline Imipenem & $20(100 \%)$ & $18(100 \%)$ & $14(93.33 \%)$ & $10(100 \%)$ \\
\hline Meropenem & $20(100 \%)$ & $18(100 \%)$ & $15(100 \%)$ & $10(100 \%)$ \\
\hline Ofloxacin & $17(85 \%)$ & $15(83.33 \%)$ & $15(100 \%)$ & $8(80 \%)$ \\
\hline Vancomycin & NT & NT & NT & NT \\
\hline
\end{tabular}

Streptococcus pyogenes isolates were $100 \%$ sensitive to ampicillin, amikacin, gentamicin, and vancomycin. Some strains of Streptococcus pyogenes showed moderate sensitivity to azithromycin (83.33\%), meropenem (83.33\%) and ciprofloxacin $(66.66 \%)$ but $100 \%$ resistant to amoxicillin and cefoperazone.

Isolates of enterococcus species exhibited high sensitivity to four antibiotics, amikacin (100\%), vancomycin (100\%), 
ciprofloxacin (50\%) and gentamicin (50\%), while they were resistant to five antibiotics, ampicillin, ceftazidime, cefoperazone, imipenem and meropenem. Tables $2 \& 3$.

\begin{tabular}{|cccc|}
\hline Antibiotics & $\begin{array}{c}\text { Staph. aureus } \\
\mathbf{N = 9}\end{array}$ & $\begin{array}{c}\text { Streptococcus } \\
\text { pyogenes } \mathbf{N}=\mathbf{6}\end{array}$ & $\begin{array}{c}\text { Enterococcus } \\
\text { spp N =2 }\end{array}$ \\
Ampicillin & 0 & $6(100 \%)$ & 0 \\
Amoxicillin & $2(22.22 \%)$ & 0 & $\mathrm{NT}$ \\
Amikacin & $8(88.88 \%)$ & $6(100 \%)$ & $2(100 \%)$ \\
Azithromycin & $9(100 \%)$ & $5(83.33 \%)$ & $\mathrm{NT}$ \\
Ciprofloxacin & $7(77.77 \%)$ & $4(66.66 \%)$ & $1(50 \%)$ \\
Ceftazidime & $5(55.55 \%)$ & $1(16.66 \%)$ & 0 \\
Cefoperazone & $9(100 \%)$ & 0 & 0 \\
Gentamicin & $4(44.44 \%)$ & $6(100 \%)$ & $1(50 \%)$ \\
Imipenem & $\mathrm{NT}$ & $\mathrm{NT}$ & 0 \\
Meropenem & $9(100 \%)$ & $5(83.33 \%)$ & 0 \\
Ofloxacin & $1(11.11 \%)$ & $1(16.66 \%)$ & $\mathrm{NT}$ \\
Vancomycin & $9(100 \%)$ & $6(100 \%)$ & $2(100 \%)$ \\
\hline \multicolumn{4}{|c|}{ Table 3. Comparative Sensitivity (Percentage of } \\
Gram-Positive Bacteria) to Different Antibiotics \\
\hline \multicolumn{4}{r}{}
\end{tabular}

\section{DISCUSSION}

This study revealed the major infection sites, different types of pathogenic bacteria in ICU and their sensitivity to commonly used antibiotics. Nosocomial infections cause significant morbidity and mortality among patients. The rate of nosocomial infection among ICU patients due to Gramnegative bacteria is significantly higher than that recorded by Gram- positive bacteria (78.75 \%, $21.25 \%$ respectively). These results resemble the results obtained by ${ }^{23}$ Gramnegative bacteria and achieved high percentage incidence $(89.76 \%)$ in the ICU. Different bacterial isolates were isolated from different infection sites, 30 isolates (37.5\%) were from tracheal aspirate; 20 (25\%) isolates from urine; $11(13.75 \%)$ isolates from blood samples; 9 (11.25\%) isolates from postsurgical wound swabs; 6 (7.5\%) isolates from central venous catheters and $4(5 \%)$ isolates from pus swabs.

Klebsielleae pneumoniae 20 (25\%) was the most prevalent bacterial organism from all clinical specimens followed by Pseudomonas aeruginosa 18 (22.5 \%), Acinetobacter baumannii 15 (18.75\%), Escherichia coli 10 (12.50 \%), Staphylococcus aureus 9 (11.25\%), Streptococcus pyogenes 6 $(7.50 \%)$ and enterococcus spp.2 (2.50\%). These results were nearly close to the results recorded by ${ }^{4} \mathrm{WHO}$ found out that the most prevalent Gram-negative bacteria were K. pneumonia (26.8\%), E. coli (22\%) and A. baumannii (17.3\%) while $S$. aureus as a Gram-positive bacterium was (10\%) in the ICU. In Asian countries, the most common pathogen isolated from patient specimens in ICU were klebsiella, pseudomonas, E. coli, and staphylococcus. ${ }^{24}$ In a similar study in ICU, Gram-negative bacteria were the predominant and included Escherichia coli (20 \%), Klebsiella pneumoniae (14.3 \%), Acinetobacter baumannii (13.7 \%), Pseudomonas aeruginosa (9 \%), Enterobacter aerogenes (5.1\%) and gram positive bacteria include Coagulase negative staphylococci (13.0\%), MRSA (7.1 $\%)$, Streptococcus pneumoniae (2.0\%) and enterococcus faecalis. $(3.1 \%)^{25}$

K. pneumoniae (40\%), A. baumannii (23.33\%) and $P$. aeruginosa (16.66\%) were the commonest organisms isolated from the tracheal aspirate. These results were nearly close to the results recorded by ${ }^{26}$ science. K. pneumonia (30.2\%) and $P$. aeruginosa $(28.7 \%)$ were the commonest organisms followed by $E$. coli $(19.4 \%)$ isolated from respiratory tract specimens. Klebsiella, 44 (53 \%) was the most common organism from the respiratory tract. ${ }^{27}$

E. coli $(40 \%)$ and K. pneumonia (25\%) were the most prevalent organisms isolated from urine specimens. Similarly, K. pneumoniae (25\%), E. coli. (23.5\%) and P. aeruginosa (16.2 \%) were isolated from UTIs. ${ }^{28}$ Gram-negative bacteria, E. coli, $K$. pneumonia and $P$. aeruginosa were the commonest organisms isolated from UTIs in Pakistan. ${ }^{29}$ Streptococcus pyogenes (36.36\%) and Staphylococcus aureus (27.27\%) were the most frequent organisms isolated from blood samples. The rate of positive blood cultures for coagulase negative Staphylococci (CNS) were $41 \%, 39 \%, 40 \%, 41 \%, 39 \%, 39 \%$, $36 \%$ and $40 \%$, respectively, from 2001 to 2008.30 The percentage incidence for both $E$. coli and $P$. aeruginosa was (31.6\%), (31.6\%), Streptococcus pneumoniae (21.1\%), and K. pneumonia (10.5\%) were isolated from blood samples. The most commonly isolated bacteria were Pseudomonas spp. In blood stream infection. ${ }^{31}$

The most common organism isolated from post-surgical wound swabs and pus swabs was Pseudomonas aeruginosa with percentage incidence (55.55\% and $50 \%$, respectively). Whereas the bacterial organisms (Streptococcus pyogenes, Staphylococcus aureus, Acinetobacter baumannii and Pseudomonas Aeruginosa) which were isolated from central venous catheter tips achieved the same percentage incidence (25\%). Group D streptococcus was the commonest isolate from wound swabs and pus swabs followed by coagulase negative staphylococcus. Acinetobacter species and Pseudomonas aeruginosa were also commonly isolated from wound and pus swabs. The pattern of distribution of Stenotrophomonas maltophilia, Pseudomonas aeruginosa and acinetobacter spp. isolated from CVP tips. ${ }^{32}$

The prevalent Klebsiella Pneumoniae isolates showed high, moderate and low sensitivity rates against all antibiotics tested in this study. The most effective antibiotics were imipenem and meropenem, the sensitivity of $K$. Pneumoniae isolates, $100 \%$, followed by ciprofloxacin, cefoperazone ofloxacin, amikacin and gentamicin, the susceptibility of $\mathrm{K}$. Pneumoniae was (95\%, $90 \%, 85 \%, 75 \%$ and $50 \%$, respectively).

Few isolates of K. pneumonia showed very low sensitivity rates with amoxicillin, ceftazidime and ampicillin (20\%, $15 \%$ and $10 \%$, respectively). In another study Klebsiella pneumonia isolates showed high susceptibility to imipenem, meropenem and sulphameth-trimeth $(74.0 \%, 67.8 \%$, and $67.8 \%$, respectively) but exhibited high resistant to ampicillin, ampicillin + sulbactam, amoxicillin - clavulanic and amikacin (96.4\%, 82.2\%, $71.5 \%$ and $37 \%$, respectively). ${ }^{33}$

The highest resistance of Klebsiella pneumoniae was observed against $\beta$-lactams $(100 \%$ isolates were resistant to ampicillin and $94 \%$ to ampicillin / sulbactam, $93 \%$ of isolates were resistant to ceftriaxone, $89 \%$ to cefotaxime, $94 \%$ to ceftazidime and cefepime), However, it was relatively less resistant to piperacillin-tazobactam (47\%), gentamicin (44 $\%)$ and amikacin (22 \%). ${ }^{34}$

In our study Pseudomonas aeruginosa isolate achieved high sensitivity rates against six antibiotics. The most effective antibiotics were imipenem, meropenem and gentamicin. The sensitivity of Pseudomonas aeruginosa isolates was $100 \%$, followed by amikacin, ofloxacin, and gentamicin. The susceptibility of Pseudomonas aeruginosa were $88 \%$, $83.33 \%$ and $77.7 \%$, respectively. But it was resistant to amoxicillin, 
ceftazidime and cefoperazone. These results were nearly close to the results of 35 Pseudomonas aeruginosa and showed high sensitivity to gentamicin and ciprofloxacin $(100 \%$ and $93 \%$, respectively).

Pseudomonas aeruginosa showed high sensitivity to tomopenem and amikacin (89.54 \% and $64.66 \%$, respectively) and showed low sensitivity against ciprofloxacin (20.92\%) and ceftazidime. (27.45\%). ${ }^{31}$

According to the study, $P$. aeruginosa recorded high sensitivity towards amikacin (90.5 \%) and moderate susceptibility towards imipenem, gentamicin, cefotaxime, ofloxacin, ciprofloxacin $(33 \%, 30 \%, 25.7 \%$ and $24.3 \%$, respectively). $P$. aeruginosa achieved low sensitivity towards ceftriaxone and ceftazidime (16.8\% and $14.9 \%$, respectively).

Acinetobacter baumannii achieved high sensitivity (100\%) towards most of the tested antibiotics amoxicillin, gentamicin, meropenem and ofloxacin, followed by ciprofloxacin, imipenem and ampicillin (93.33\%, $93.33 \%$ and $86.66 \%$, respectively). Acinetobacter showed low sensitivity against amikacin $(13.33 \%)$. These results were nearly close to the results of ${ }^{34}$ science Acinetobacter baumannii and showed high sensitivity to imipenem and ampicillin (92.6\% and $80.6 \%$ ). $A$. baumannii displayed the lowest level of sensitivity with $3 \%, 4$ $\%, 5 \%, 8 \%$ and $13 \%$ to ciprofloxacin, meropenem, imipenem, ceftazidime and gentamicin.

E. coli displayed the high levels of sensitivity with $100 \%$, $90 \%, 80 \%$ and $70 \%$ sensitivity to imipenem, meropenem, amikacin, gentamicin, ofloxacin and ciprofloxacin. E. coli recorded low sensitivity towards amoxicillin (20\%). These results were nearly close to the results recorded by ${ }^{34} E$. coli that achieved high sensitivity to amikacin, meropenem, gentamicin and ciprofloxacin $(100 \%, 96 \%, 93 \%$ and $91 \%$, respectively). In another study $E$. coli recorded high susceptibility to imipenem, amikacin and gentamicin (84.9\%, $75.5 \%$ and $50.9 \%$, respectively) and showed low sensitivity to ceftazidime, ofloxacin and ciprofloxacin $(28.3 \%, 24.5 \%$ and $24.5 \%$, respectively).

Staphylococcus aureus showed considerable sensitivity to azithromycin, cefoperazone, meropenem, vancomycin, amikacin, ciprofloxacin, ceftazidime and gentamicin (100\%, $100 \%, 100 \%, 100 \%, 88.88 \%, 77.77 \%, 55,55 \%$ and 44.44 $\%$, respectively). S. aureus recorded low sensitivity to amoxicillin and ofloxacin $(22.22 \%$ and $11.11 \%$ respectively.)

These results were nearly close to the results of study where $S$. aureus exhibited high sensitivity to vancomycin, amikacin, ofloxacin, cefotaxime and imipenem (100\%, $92.3 \%$, $61.6 \%, 53.8 \%$ and $46.2 \%$ respectively) but recorded low sensitivity to ciprofloxacin (38.5\%) and gentamicin (23.1\%).

S. aureus showed good sensitivity to vancomycin (100\%), ciprofloxacin (88\%) and gentamicin (73\%). ${ }^{35}$

Streptococcus pyogenes exhibited high sensitivity to different types of antibiotics which include ampicillin, amikacin, gentamicin, vancomycin, azithromycin, meropenem and ciprofloxacin $(100 \%, 100 \%, 100 \%, 100 \%, 83.33 \%$, $83.33 \%$ and $66.6 \%$, respectively). S. pyogenes recorded very low sensitivity to ceftazidime and ofloxacin (16.6\%). These results are in agreement with the previous study ${ }^{36}$ since $S$. pyogenes achieved high sensitivity $100 \%$ to ampicillin, gentamicin and vancomycin. In contrast $S$. pyogenes recorded high resistant (100\%) to ampicillin, gentamycin, ciprofloxacin and amikacin.
Enterococcus spp. recorded high sensitivity $100 \%$ to amikacin and vancomycin but exhibited moderate sensitivity (50 \%) to ciprofloxacin and gentamicin. These results in similar to the results recorded by ${ }^{37}$ the sensitivity of enterococcus spp. was $100 \%$ to amikacin, ciprofloxacin, vancomycin and imipenem. Also, the sensitivity to gentamicin was $50 \%$. In another study enterococcus spp. recorded high sensitivity to vancomycin (100\%) and moderate sensitivity to gentamicin (33\%). ${ }^{37}$

\section{CONCLUSIONS}

Nosocomial infection is a global problem in ICUs of different hospitals and is a major cause of mortality in hospitals. The role of nursing care in the prevention of nosocomial infection has proven vital due to the emergence and spreading of different pathogenic bacteria. Close interactions between health care providers can save many more lives. We studied the isolation, identification, and detection of the sensitivity pattern of different pathogenic bacteria isolated from intensive care unit under the action of infection control. The choice of the antibiotics in ICU patients is usually empirical. The ongoing surveillance of antibiotic susceptibility patterns of predominant bacteria is an essential primary effort to guide the clinician in choosing empirical or directed therapy appropriately, especially in the ICU setting. This study showed that the antibiotic sensitivity pattern of most common pathogens in ICU may save the lives of the patients and prevent the spreading of hospital-acquired infection. This recorded data will be useful for healthcare professionals in deciding the suitable antibiotics for different pathogenic bacteria that emerge in critical areas like ICU.

Data sharing statement provided by the authors is available with the full text of this article at jemds.com.

Financial or other competing interests: None.

Disclosure forms provided by the authors are available with the full text of this article at jemds.com.

\section{REFERENCES}

[1] Drees M, Pineles L, Harris AD, et al. Variation in definitions and isolation procedures for multidrug-resistant gramnegative bacteria: a survey of the Society for Healthcare Epidemiology of America Research Network. Infect Control Hosp Epidemiol 2014;35(4):362-6.

[2] Siegel JD, Rhinehart E, Jackson M, et al. Management of multidrug-resistant organisms in health care settings, 2006. Am J Infect Control 2007;35(10 Suppl 2):S165-93.

[3] Siegel JD, Rhinehart E, Jackson M, et al. 2007 guideline for isolation precautions: preventing transmission of infectious agents in health care settings. Am J Infect Control 2007;35(10 suppl 2):S65-164.

[4] Tacconelli E, Cataldo MA, Dancer SJ, et al. ESCMID guidelines for the management of the infection control measures to reduce transmission of multidrug-resistant gram-negative bacteria in hospitalized patients. Clin Microbiol Infect 2014;20(suppl 1):1-55. 
[5] Moodley P, Whitelaw A. The pros, cons and unknowns of search and destroy for carbapenem-resistant enterobacteriaceae. Curr Infect Dis Rep 2015;17(6):483.

[6] Harris AD, McGregor JC, Furuno JP. What infection control interventions should be undertaken to control multidrugresistant gram-negative bacteria? Clin Infect Dis 2006;43(Suppl 2):S57-61.

[7] Proposal for a Council Recommendation on patient safety, including the prevention and control of healthcare associated infections $\{\mathrm{COM}(2008) 836$ final $\}$ SEC(2008) $3004\}\left\{\right.$ SEC(2008) 3005\}. EC Brussels: $20^{\text {th }}$ January 2009.

[8] Hoque l, Kamal MM, Ahmed Z. Isolation, identification and antimicrobial sensitivity pattern of bacterial isolates from tracheal aspirate of ICU patients of Central Dhaka, Bandladesh. Int J Res Appl Nat Soc Sci 2013;1(7):11-6.

[9] Vincent DL, Bihari DJ, Suter PM, et al. The prevalence of nosocomial infection in intensive care units in Europe. Results of the European prevalence of infection in Intensive Care (EPIC) study. EPIC International Advisory Committee. JAMA 1995;274(8):639-44.

[10] Tennant I, Harding H, Nelson M, et al. Microbial isolates from patients in an intensive care unit and associated risk factors. West Indian Med J 2005;54(4):225-31.

[11] Weber DJ, Raasch R, Rutala WA. Nosocomial infections in the ICU: the growing importance of antibiotic-resistant pathogens. Chest 1999;115(3 Suppl):34S-41S.

[12] Richards M, Thursky K, Buising K. Epidemiology, prevalenc andsites of infections in intensive care units. Semin Respir Crit Care Med 2003;24(1):3-22.

[13] Ylipalosaari P, Ala-Kokko TI, Laurila J, et al. Intensive care acquired infection is an independent risk factor for hospital mortality: a prospective cohort study. Crit Care 2006;10(2):R66.

[14] Gandhi S, Ranjan KP, Ranjan N, et al. Incidence of neonatal sepsis in tertiary care hospital: an overview. Int J Med Sci Public Health 2013;2(3):548-52.

[15] Aftab R, Iqbal I. Bacteriological agents of neonatal sepsis in NICU at Nishtar Hospital Multan. J Coll Physicians Surg Pak 2006;16(3):216-9.

[16] Hafsa A, Fakruddin M, Hakim MA, et al. Neonatal bacteremia in a neonatal intensive care unit: analysis of causative organisms and antimicrobial susceptibility. Bangladesh Journal of Medical Science 2011;10(3):18794.

[17] Nahar BS, Afroza S, Roy S, et al. Neonatal sepsisin a tertiary care hospital: evaluation of causative agents and antimicrobial susceptibilities. Bangladesh J Child Health 2013;37(1):14-7.

[18] Kollef MH, Fraser VJ. Antibiotic resistance in the intensive care unit. Ann Intern Med 2001;134(4):298-314.

[19] Shankar PR, Partha P, Dubey AK, et al. Intensive care unit drug utilization in a teaching hospital in Nepal. Kathmandu Univ Med J (KUMJ) 2005;3(2):130-7.

[20] Tripathi KD. Essentials of Medical Pharmacology. $6^{\text {th }}$ edn New Delhi: Jaypee Brothers 2009:667-81.

[21] Shlaes DM, Gerding DN, John JF Jr, et al. Society for healthcare epidemiology of America and infectious diseases society of america joint committee on the prevention of antimicrobial resistance: guidelines for the prevention of antimicrobial resistance in hospitals. Clin Infect Dis 1997;25(3):584-99.
[22] Sharma PR, Barman P. Antimicrobial consumption and impact of reserve antibiotic indent form in an intensive care unit. Indian J Pharmacol 2010;42(5):297-300.

[23] Brink A, Moolman J, Da Silva MC, et al. Antimicrobial susceptibility profile of selected bacteraemic pathogens from private institutions in South Africa. S Afr Med J 2007;97(4):273-9.

[24] Radji M, Fauziah S, Aribinuko N. Antibiotic sensitivity pattern of bacterial pathogens in the intensive care unit of Fatmawati Hospital, Indonesia. Asian Pac J Trop Biomed 2011;1(1):39-42.

[25] Sarvepalli AK, Dharana PK. Clinical profile, bacterial profile and outcomes in an intensive care unit of a tertiary care hospital in South India: one year study. Int J Adv Med 2017;4(1):156-61.

[26] Lorente L, Blot S, Rello J. New issues and controversies in the prevention of ventilator-associated pneumonia. Am J Respir Crit Care Med 2010;182(7):870-6.

[27] Akter T, Murshed M, Begum T, et al. Antimicrobial resistance pattern of bacterial isolates from intensive care unit of a tertiary care hospital in Bangladesh. Bangladesh J Med Microbiol 2014;8(1):07-11.

[28] Rizvi MF, Hasan Y, Memon AR, et al. Pattern of nosocomial infection in two intensive care units of a tertiary care hospital in Karachi. J Coll Physicians Surg Pak 2007;17(3):136-9.

[29] Izhar M, Khan S, Naqvi A. Anti-microbial resistance among gram-negative bacteria prevalent in intensive care units. Pak J Surg 2001;17(3):23-7.

[30] Cortes JA, Leal AL, Montanez AM, et al. Frequency of microorganisms isolated in patients with bacteremia in intensive care units in Colombia and their resistance profiles. Braz J Infect Dis 2013;17(3):346-52.

[31] Raval PN, Patel PG, Patel BV, et al. Microbiological surveillance of intensive care units in a tertiary care teaching hospital-Western India. International Journal of Microbiology Research 2012;4(7):270-4.

[32] Nicholson AM, Ledgister S, Williams T, et al. Distribution of nosocomial organisms and their resistance patterns in theintensive care unit of the university hospital of the West Indies, Kingston, Jamaica. West Indian Med J 2009;58(2):142-8.

[33] El-Shiekh H, Gaafar M, Yosri M, et al. Study of bacteria causing septicemia in neonatal intensive care unit. Egyptian Journal of Medical Microbiology 2016;25(1):3744.

[34] Injac V, Batranović U, Matijasevic J, et al. Etiology and resistance patterns of bacteria causing ventilatoras sociatedpneumonia in a respiratory intensive care unit. Vojnosanitetski Pregled 2017;74(10):954-62.

[35] Greatorex B, Frca G, Oosthuizen M. Organisms cultured and resistance patterns seen in a secondary referral centre ICU and burns unit. South Afr J Crit Care 2015;31(1):12-5.

[36] Pandita N, Wasim S, Bhat NK, et al. Identification of the bacterial isolates in neonatal septicaemia and their antimicrobial susceptibility in a tertiary care hospital in Uttarakhand, India: a retrospective study. Int J Contemp Pediatr 2016;3(1):200-5.

[37] Shrestha S, Shrestha NC, Singh SD, et al. Bacterial isolates and its antibiotic susceptibility pattern in NICU. Kathmandu Univ Med J (KUMJ) 2013;11(41):66-70. 